\title{
Complete Response of Linear Circuits to Periodic Nonsinusoidal Input in MATLAB
}

\author{
IvetaTomčíková \\ Technical University in Košice, Department of Theoretical and Industrial Electrical Engineering, Košice, Slovakia, \\ e-mail: Iveta.Tomcikova@tuke.sk
}

\begin{abstract}
The paper deals with the proposal for finding the complete response of dynamic linear circuits to a periodic nonsinusoidal input in the MATLAB environment. A very powerful tool for solving the given problem is to transform the circuits directly into the complex frequency domain using the Laplace transform and then apply the sparse tableau analysis technique to solve them. Applying above-mentioned methods in the MATLAB environment, it is not difficult to find the complete response of dynamic linear circuits to the periodic input.
\end{abstract}

Keywords - complete response, transient response, steadystate response, sparse tableau analysis, Laplace transform, MATLAB

\section{INTRODUCTION}

The problem of finding the complete response of dynamic circuits to a periodic nonsinusoidal input can be solved in the time or in the complex frequency domain. In both cases, the given problem is time-consuming activity that involves a lot of effort and skills in mathematics. In the time domain, circuit equations take the form of integrodifferential equations with the periodic function on the right-hand sides. In the complex frequency domain, the circuit equations take form of algebraic equations with the Laplace transform of the periodic function.

MATLAB enables to reduce greatly the effort demanded to solve the problem of finding the complete response of dynamic circuits in the complex frequency domain made by hand. In the MATLAB environment, it is possible to formulate the circuit equations in a systematic and automatic way using the sparse tableau analysis, to take the Laplace transform and the Laplace inverse transform, and to combine this procedure with its symbolic computation.

\section{FINDING THE COMPLETE RESPONSE OF CIRCUIT TO PERIODIC NONSINUSOIDAL INPUT}

\section{A. Circuit Equations}

In order to describe the circuit it is necessary to compose the circuit equations. The set of the circuit equations consists of two sets of equations: the equations that represent the topology of the circuit and the equations that represent the type of the circuit elements.

The first set of the circuit equations depicts how the circuit elements are connected to one another in the circuit. These equations are called the connection equations.

The second set of the circuit equations describes the voltage-current relationship of circuit elements and they are called the element equations.
Many systematic methods are available for the mathematical description of the circuits, but better ones are those methods, which enable to formulate the circuit equations in a systematic and automatic way, e.g. modified nodal analysis and sparse tableau analysis, because they can be used for computer simulation of circuits.

\section{B. Sparse Tableau Analysis}

The sparse tableau analysis offers not only systematic and automatic approach for assembling the circuit equations but also their subsequent solution provides the currents through all elements, the voltages across all elements and all nodal voltages simultaneously.

In general, the circuit equations formulated by the sparse tableau analysis procedure take form of a set of simultaneous nonlinear first-order differential equations in the time domain. For a purely resistive and linear circuit they take form of a set of simultaneous linear algebraic equations. When the linear circuit is transformed into its complex frequency domain equivalent using the Laplace transform, then it can be dealt with it as if it is consisted of sources and resistors only, because the passive elements can be represented as the series connection of two circuit elements: a complex frequency domain impedance, which can be regarded as generalized resistance, and the source of initial condition. In this case, the circuit equations take form of a set of simultaneous linear algebraic equations in the complex frequency domain.

Let us consider a linear dynamic circuit, for which the connection and parameters of two-terminal elements, forming the circuit, are given. Let the number of the circuit elements (branches) be $M$ and the number of circuit nodes $N$. The sparse tableau analysis procedure of setting up the circuit equations in the complex frequency domain consists of the two steps: the step of composing the connection equations and the step of composing the element equations.

The connection equations are obtained by applying Kirchhoff's laws to the circuit, which leads to two sets of linear algebraic equations in terms of the element currents and element voltages. The set of connection equations must be linearly independent. The first set of the connection equations can be expressed [1]

$$
\mathbf{A} \mathbf{i}(s)=\mathbf{0},
$$

where $\mathbf{i}(s)$ is a vector of the type $(M, 1)$, whose elements are the complex frequency-domain representation of circuit element currents $I_{1}(s), I_{2}(s), \ldots, I_{M}(s) ; \mathbf{A}$ is the node versus branch reduced-incidence matrix of the type $(N-1, M)$, having the coefficients $+1,-1,0$. 
The second set of the connection equations can be expressed in terms of the element voltages and the node voltages as [1]

$$
\mathbf{u}(s)=\mathbf{A}^{\mathrm{T}} \mathbf{v}(s),
$$

where $\mathbf{u}(s)$ is a vector of the type $(M, 1)$, whose elements are the complex frequency-domain representation of the circuit element voltages $U_{1}(s), U_{2}(s), \ldots, U_{M}(s) ; \mathbf{v}(s)$ is a vector of the type $(n-1,1)$, whose elements are the complex frequency-domain representation of the node voltages $V_{1}(s), V_{2}(s), \ldots, V_{N-1}(s) ; \quad \mathbf{A}^{\mathrm{T}}$ is the transposed matrix A .

The element equations express the voltage-current characteristics of the circuit elements that are the relation between the voltage across and the current through the circuit element.

Let us consider that the $k$ th circuit element is described in the complex frequency domain by the voltage-current relationship having one of the following forms: $U_{k}(s)=F_{k}(s), \quad I_{k}(s)=F_{k}(s), \quad U_{k}(s)=c_{k l}^{u u} U_{l}(s)$, $U_{k}(s)=c_{k l}^{u i} I_{l}(s), I_{k}(s)=c_{k l}^{i u} U_{l}(s)$ or $I_{k}(s)=c_{k l}^{i i} I_{l}(s)$, $U_{k}(s)-Z_{k}(s) I_{k}(s)= \pm U_{k}^{(\mathrm{ic})}(s)$, where $F_{k}(s)$ is the Laplace transform of voltage/current of independent voltage/current sources, $c_{k l}^{u u}, c_{k l}^{u i}, c_{k l}^{i u}, c_{k l}^{i i}$ are gains of linear dependent voltage/current sources, $Z_{k}(s)$ is the complex frequency domain impedance of linear passive element, $U_{k}^{(\text {ic) }}(s)$ is the source of initial condition in the complex frequency domain. Then the element equations can be expressed [1]

$$
\mathbf{K}^{(u)}(s) \mathbf{u}(s)+\mathbf{K}^{(i)}(s) \mathbf{i}(s)=\mathbf{f}(s),
$$

where $\mathbf{K}^{(u)}(s), \mathbf{K}^{(i)}(s)$ are matrices of the type $(M, M)$, containing the complex frequency-domain representation of coefficients that define the linear voltage-current relationships for the circuit elements unambiguously; $\mathbf{f}(s)$ is a vector of the type $(M, 1)$, which contains the complex frequency-domain representation of the independent voltage and current sources parameters.

Assembling Eqs. (1), (2), and (3), the sparse tableau equations are constituted. It is convenient to rewrite the sparse tableau equations as a single matrix equation [1]

$$
\mathbf{T}(s) \mathbf{x}(s)=\mathbf{w}(s),
$$

where $\mathbf{T}(s)$ is a square matrix of the type $(2 M+N-1,2 M+N-1)$, called the sparse tableau matrix; $\mathbf{x}(s)$ is a vector of the type $(2 M+N-1,1)$, which contains unknown variables $\mathbf{i}(s), \mathbf{u}(s), \mathbf{v}(s) ; \mathbf{w}(s)$ is a vector of the type $(2 M+N-1,1)$ containing zero vectors of appropriate dimensions and the vector $\mathbf{f}(s)$.

After solving Eq. (4), the unknown variables are obtained, but only the first $2 M$ elements of the vector $\mathbf{x}(s)$ represent the element currents and voltages.

\section{Description of Periodic Nonsinusoidal Input in the}

Time Domain and in the Frequency Domain

Let $h(t)$ is a periodic input, i.e. a periodic function of real variable $t$ having the period $T>0$ that satisfies the following conditions simultaneously [2], [3]:

- for all $t<0: h(t)=0$,

- for all $t \geq 0: h(t)$ must be piecewise continuous,

- $\quad$ the magnitude of $h(t)$ must be $|h(t)|<\beta \exp (\alpha t)$ for all positive $t$, where $\beta, \alpha$ are constants.

Let $h_{T}(t)$ be a pulse, which is identical to the input $h(t)$ at the time interval $\langle 0, T)$, and zero out of the interval. Repeating the pulse $h_{T}(t)$ periodically in the time points $n T, n=0,1,2, \ldots$, the input $h(t)$ is created and it can be expressed for $t \geq 0$ as the sum of infinite number of time-shifted finite pulses [3]

$$
h(t)=\sum_{n=0}^{\infty} h_{T}(t-n T) .
$$

Applying the Laplace transform to the equation (5) together with the linearity property and time shifting property, the geometric series having a quotient $\exp (-s T)$ is obtained

$$
H(s)=\sum_{n=0}^{\infty} H_{T}(s) \cdot \exp (-s . n T),
$$

where $H_{T}(s)$ is the Laplace transform of the pulse $h_{T}(t)$.

The geometric series converges for $|\exp (-s T)|<1$, i.e. for $\operatorname{Re}\{s\}>0$, therefore the Laplace transform $H(s)$ of the periodic input $h(t)$ is [3]

$$
H(s)=H_{T}(s) /(1-\exp (-s T)) .
$$

\section{Procedure for Finding Circuit Complete Response to Periodic Nonsinusoidal Input}

Let us consider the linear dynamic circuit with zero initial conditions, which is excited by a single periodic nonsinusoidal input.

In the complex frequency domain, the circuit element currents and voltages have a form (solution of the sparse tableau equations):

$$
X_{k}(s)=\frac{P_{k}(s)}{Q(s)} H(s), \quad \text { for } k=1,2, \ldots, 2 M,
$$

where $P_{k}(s), Q(s)$ are $M_{P k}$-th degree, $M_{Q}$-th degree polynomials in $s$.

The complex frequency domain representation $X_{k}(s)$, $k=1,2, \ldots, 2 M$, must have poles at the points, where $\exp (-s T)=1$, and $Q(s)=0$. For simplicity, the following assumptions are made for $X_{k}(s)$, $k=1,2, \ldots, 2 M$ : 
- $\quad$ the degree of polynomials $P_{k}(s), k=1,2, \ldots, 2 M$, are less than that of the polynomials $P_{k}(s), Q(s)$, i.e. $M_{P k}<M_{Q}$,

- all real poles and real parts of all complex poles, which are the roots of $Q(s)=0$, are negative so that the circuit is stable.

In order to find the complete response of the circuit to the periodic nonsinusoidal input it is necessary [4]:

- $\quad$ to find out the poles of $X_{k}(s), k=1,2, \ldots, 2 M$, at the points, where $Q(s)=0$, i.e. to find the roots of the polynomial $Q(s)$,

- to evaluate (take the inverse Laplace transform of) $X_{k}(s), k=1,2, \ldots, 2 M$, but only for roots of the polynomial $Q(s)$; this part is the transient part $x_{k}^{\text {(trans) }}(t)$ of the complete response of the corresponding element current or voltage of given circuit for $t>0$,

- to take the inverse Laplace transform of the expression (8) where $H(s)$ is replaced with the terms of $H_{T}(s)$ that act for $t<\mathrm{T}$, in order to obtain the complete response $x_{k}^{(\text {total })}(t)$ of the corresponding element current or voltage of given circuit for $0<t<T$ (but not for $t>T$ ),

- $\quad$ to subtract the transient response $x_{k}^{\text {(trans) }}(t)$ from the complete response $x_{k}^{(\text {total })}(t)$ of the corresponding element current or voltage of given circuit at the interval $0<t<T$, in order to obtain the steady-state response $x_{k_{T}}^{\text {(steady) }}(t)$ of the corresponding element current or voltage of given circuit at the interval $0<t<T$,

$$
x_{k_{T}}^{\text {(steady) }}(t)=x_{k}^{\text {(total) }}(t)-x_{k}^{\text {(trans) }}(t), \quad 0<t<T,
$$

for $k=1,2, \ldots, 2 M$.

- the steady-state response repeats periodically, i.e.

$$
x_{k}^{\text {(steady) }}(t)=\sum_{n=0}^{\infty} x_{k_{T}}^{\text {(steady) }}(t-n T),
$$

therefore the complete response $x_{k}^{(\text {total })}(t)$ of the corresponding element current or voltage of given circuit for $t>T$ can be found by addition of the transient response $x_{k}^{\text {(trans) }}(t)$ and the steady-state response $x_{k}^{\text {(steady) }}(t)$ of the corresponding element current or voltage of given circuit for $t>T$,

$$
x_{k}^{(\text {total })}(t)=x_{k}^{(\text {steady) }}(t)+x_{k}^{(\text {trans })}(t), \quad t>T,
$$

for $k=1,2, \ldots, 2 M$

\section{EXAMPLES OF FINDING COMPLETE RESPONSE OF CIRCUIT IN MATLAB}

All steps for finding the complete response of a dynamic circuit given above can be easily executed using the program composed and run in the MATLAB environment.

The following text contains two representative examples of finding the circuit complete response to the periodic nonsinusoidal input. The outputs of the examples are graphical representations of the circuit responses.

\section{A. Complete Response of Series RL Circuit to Saw-Tooth} Wave

The series $R L$ circuit (Fig. 1 - left) with the initial condition equal to zero is excited by the periodic saw-tooth wave shown in Fig. 1 (right), which is applied at time $t=0$. The given input has the following parameters: the amplitude $U$ and the period $T$.

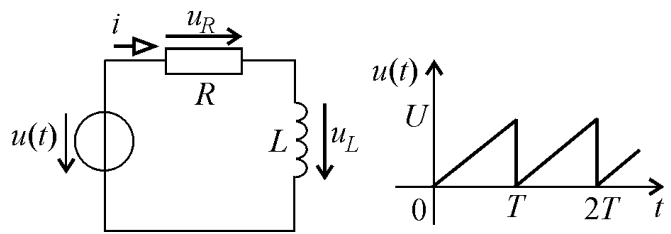

Fig. 1. Series $R L$ circuit (left) and saw-tooth wave input (right) Example A.

The aim is to find the complete response of the circuit to the given input using the program that is an implementation of the proposed procedure in the MATLAB environment.

The first input data for the program are the parameters of the circuit elements. The circuit equations are constituted automatically using the sparse tableau analysis.

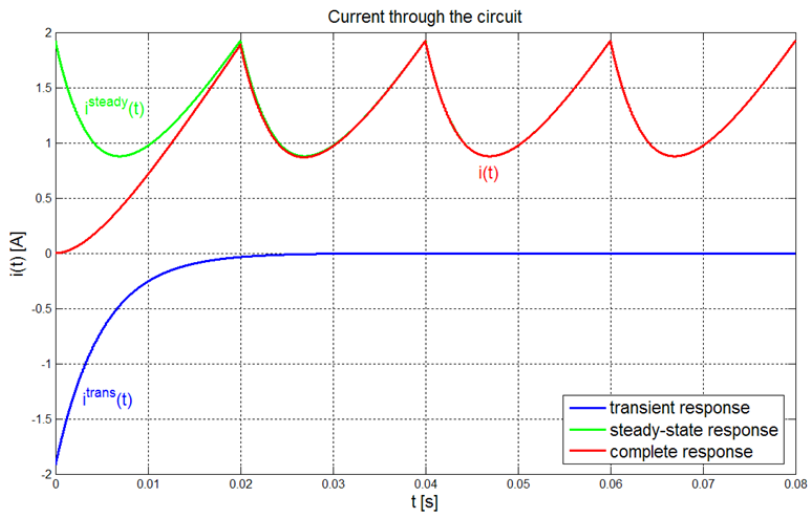

Fig. 2. MATLAB plot of the current versus time of the circuit shown in Fig. 1.

After executing all program steps according to the proposed procedure in the MATLAB environment, the results of Example A are obtained.

The corresponding graphical representations of all responses of the circuit are given for the following values of the circuit parameters: $R=20 \Omega, L=0.1 \mathrm{H}, U=50 \mathrm{~V}$, $T=0.02 \mathrm{~s}$, and they are depicted in Figs.2, 3, and 4 . 


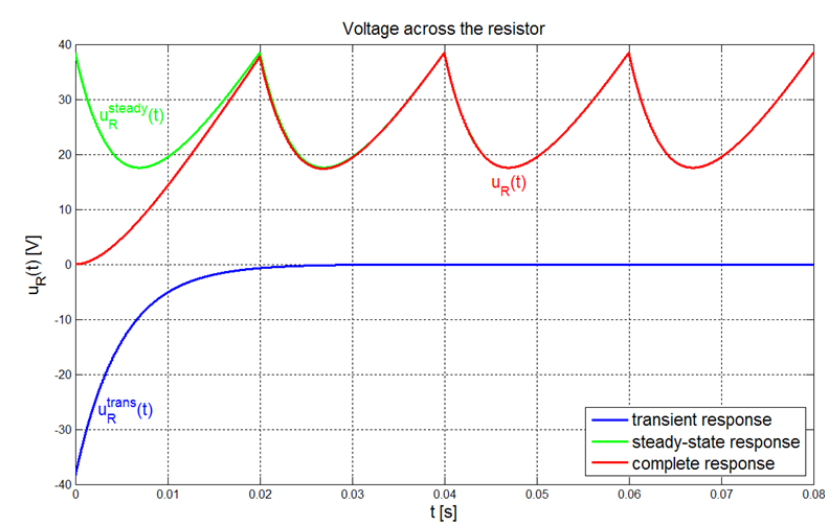

Fig. 3. MATLAB plot of resistor voltage versus time of the circuit shown in Fig. 1.

In Fig. 2, the transient, steady-state and complete response of the current flowing through the given circuit is shown. In Figs. 3 and 4, the responses of the voltage across the resistor and the voltage across the inductor of the given circuit are depicted.

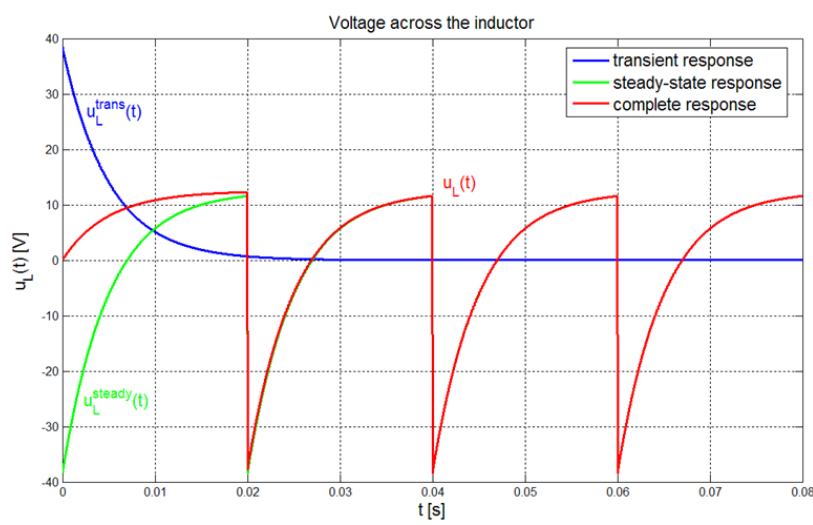

Fig. 4. MATLAB plot of inductor voltage versus time of the circuit shown in Fig. 1.

All plots indicate that after the first period the influence of the transient responses can be considered as negligible for practical purposes.

\section{B. Complete Response of Second-Order Circuit to Square Wave}

Figure 5 (left) shows a second-order circuit with the zero initial conditions. The input to the circuit is the voltage of the voltage source that is the periodic square wave having the amplitude $U$ and the period $T$, which is applied at time $t=0$. The aim is to find the complete response of the given circuit.

After running the program the results of Example $B$ are obtained. The corresponding graphical representations of all element currents and voltages are given for the following values of the circuit parameters: $R=100 \Omega$, $L=12 \mathrm{mH}, C=2 \mu \mathrm{F}, T=0.008 \mathrm{~s}, U=25 \mathrm{~V}$. They are shown in Figs. 6 to 10.
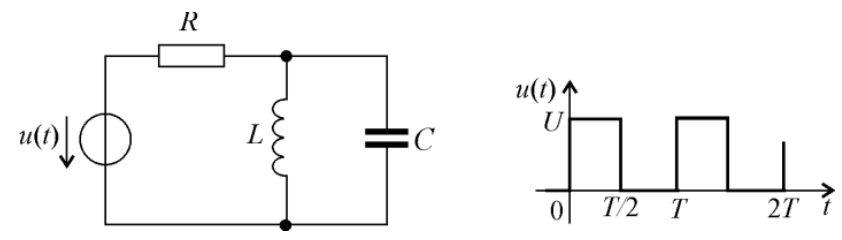

Fig. 5. The second-order circuit (left) and square wave input (right) Example B.

In the problem of Example $B$ the numeric values of the transient responses are in the range from $10^{-3}$ to $10^{-6}$ of the corresponding units and values of the complete and steady-state responses in the range from $10^{-1}$ to $10^{+2}$ of the corresponding units. Due to this, all plots shown in Figs. 6 to 10 are created with y-axes on both left and right sides of the graph. The two y-axes plots enable to display both data sets in one graph even though relative values of the data belong to quite different value ranges.

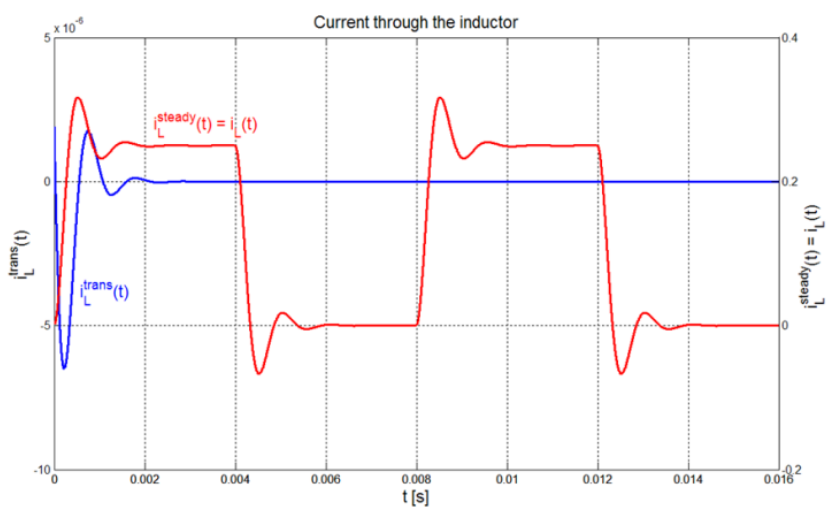

Fig. 6. MATLAB plot of the inductor current versus time of the circuit shown in Fig. 5.

In Fig. 6, the current flowing through the inductor is depicted. The plot shows the transient, steady-state, and complete responses. It is evident that the transient response of the current obtains very small values, thus the steady-state response is equal to the complete response of the inductor current and the steady-state values of the inductor current is $0 \mathrm{~A}$ and about $0.25 \mathrm{~A}$.

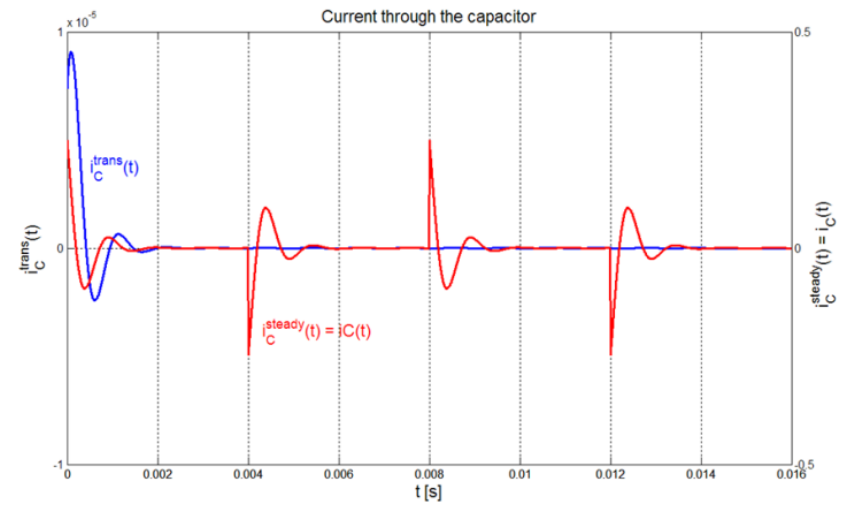

Fig. 7. MATLAB plot of the capacitor current versus time of the circuit shown in Fig. 5.

In Fig. 7, the current flowing through the capacitor is depicted. The plot shows that steady-state value of the capacitor current is $0 \mathrm{~A}$ (at steady state the capacitor acts as an open circuit). 


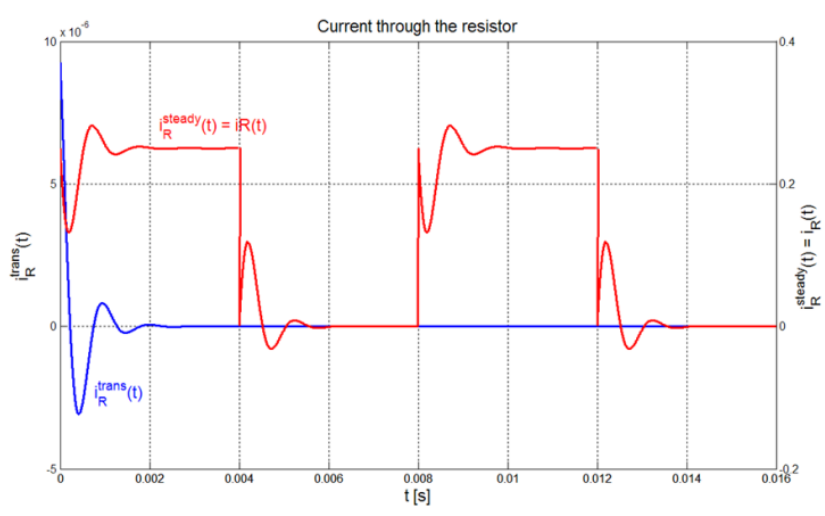

Fig. 8. MATLAB plot of the resistor current versus time of the circuit shown in Fig. 5.

In Fig. 8, the transient, steady-state, and complete responses of the current flowing through the resistor are drawn. The plot shows that steady-state values of the resistor current are $0 \mathrm{~A}$ and about $0.25 \mathrm{~A}$ (at steady state the inductor acts as a short circuit, the capacitor acts as an open circuit, thus the same current flows through the resistor and inductor).

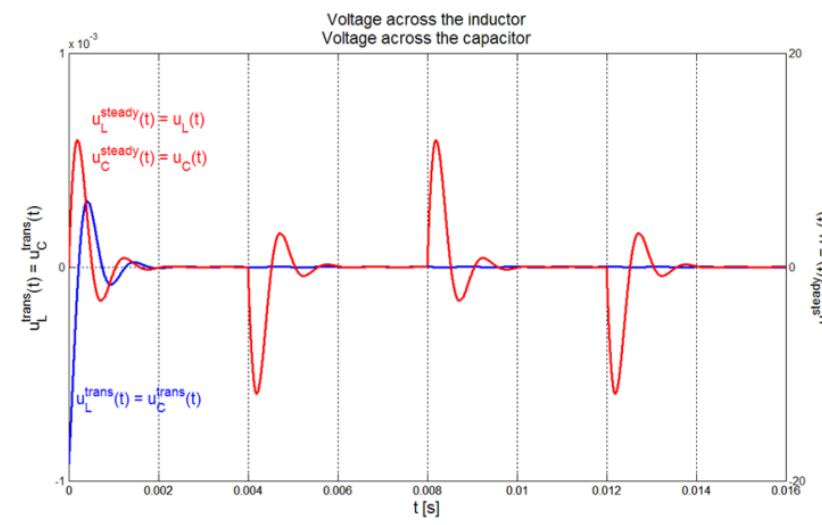

Fig. 9. MATLAB plot of the inductor and capacitor voltage versus time of the circuit shown in Fig. 5.

In Fig. 9, the voltage across the inductor and capacitor is drawn (parallel connection). The plot shows that the steady-state value of the inductor voltage is $0 \mathrm{~A}$ (at steady state the inductor acts as a short circuit).

In Fig. 10, the voltage across the resistor is depicted.

It is evident that all responses of the circuit given in Example $\mathrm{B}$ are oscillatory-type ones with decaying magnitude and the frequency of oscillation that depends on the damped resonant frequency.

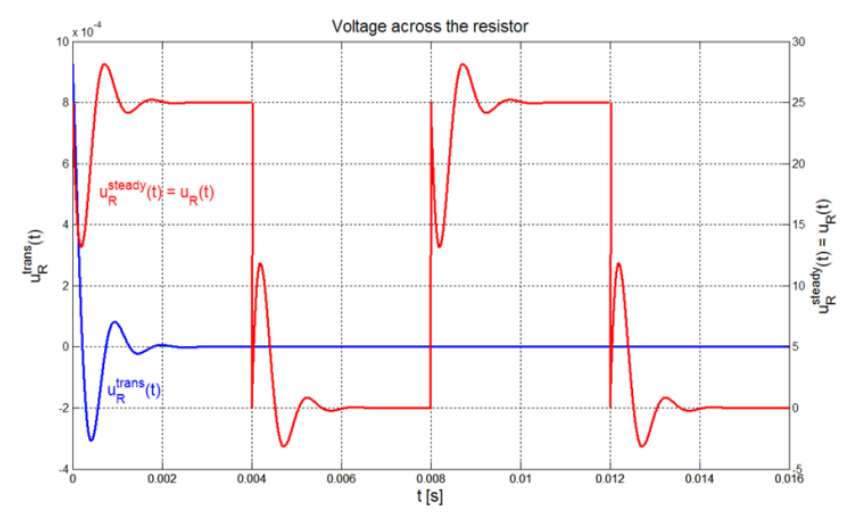

Fig. 10. MATLAB plot of the resistor voltage versus time of the circuit shown in Fig. 5.

\section{CONCLUSIONS}

The procedure for finding complete, transient, and steady-state responses of a linear circuit to the periodic nonsinusoidal input was proposed. The description of the circuit was done in the complex frequency domain using the sparse tableau analysis, thus instead of the differential equations solution, only the algebraic ones were solved.

The solution was done by running the program based on the proposed procedure composed in the MATLAB environment. Such technique has many advantages over the calculus made by hand. The first of all is the fact that it saves a lot of time and effort, because the user has not to solve the set of algebraic equations. The second advantage is the fact that he has not to take the direct and inverse Laplace transform. The third advantage consists in fact, that the program provides the graphical representation of the complete, transient, and steady-state responses for all element currents and voltages.

Since the MATLAB environment is very user-friendly, the user has not to spend time in learning software but he can spend time in learning the fundamental principles of a solved problem.

\section{REFERENCES}

[1] F. N. Najm: Circuit Simulation. JohnWiley\&Sons, Inc., 2010, pp. 13-30, ISBN 978-0-470-53871-5. http://dx.doi.org/10.1002/9780470561218.ch2

[2] R.C.Dorf, J.A.Svoboda: Introduction to Electric Circuits. John Wiley \&Sons, Inc., 2007, pp. 655-700, ISBN 978-0-471-73042-2.

[3] D. Mayer: Introduction in Theory of Electric Circuits. SNTLPrague, 1984 (in Czech), pp. 503-508.

[4] I. Mayer: Circuit Theory(Part 2). Alfa - Bratislava, 1981 (in Slovak), pp. 82-90.

[5] MATLAB -User's Guide (Mathematics, Programming, Graphics), MathWorks 2009. 